Hautarzt 2022 $\cdot 73: 638-640$ https://doi.org/10.1007/s00105-021-04922-1

Angenommen: 8. November 2021

Online publiziert: 14. Dezember 2021

(c) Der/die Autor(en) 2021

\section{Unsachgemäße Systemtherapie bei schwerer atopischer Dermatitis - fatale Langzeitschäden}

\author{
F. Abeck · N. Booken · S. Schneider
}

Klinik und Poliklinik für Dermatologie und Venerologie, Universitätsklinikum Hamburg-Eppendorf, Hamburg, Deutschland

\title{
Zusammenfassung
}

Die Therapiemöglichkeiten der schweren atopischen Dermatitis waren bis vor Kurzem sehr begrenzt und haben sich mit der Zulassung des ersten Biologikums Dupilumab Ende 2017 bis heute deutlich verbessert. Aktuell wurden mit dem Biologikum Tralokinumab sowie den Januskinase-Inhibitoren Baricitinib und Upadacitinib weitere neue Systemtherapeutika zugelassen. Dennoch gibt es Fälle, in denen es zur Nichtberücksichtigung moderner Behandlungsmöglichkeiten kommt, wie die Falldarstellung eines 28-jährigen Patienten mit schwerwiegenden Nebenwirkungen einer Langzeitbehandlung mit systemischen Glukokortikosteroiden zeigt. Neben der umfangreichen Abklärung der Folgeschäden, erfolgten die Einleitung einer leitliniengerechten Therapie mit Dupilumab sowie eine interdisziplinäre Zusammenarbeit mit Endokrinologen, Ophthalmologen, Osteologen und Ernährungsmedizinern.

\section{Schlüsselwörter}

Atopische Dermatitis · Systemtherapie · Orale Glukokortikosteroide · Nebenwirkungen

\section{Anamnese}

Wir berichten über einen 28-jährigen Patienten, bei dem seit dem ersten Lebensjahr eine atopische Dermatitis (AD) besteht. Die Familienanamnese ist positiv, da die Mutter ebenfalls von einer $A D$ betroffen ist. Bei dem Patienten liegt zusätzlich ein allergisches Asthma bronchiale vor. Das Ekzem wurde seit 6 Jahren mit systemischen Glukokortikosteroiden behandelt. Die Dosierung wurde eigenständig durch den Patienten je nach Hautbefund und Juckreiz gewählt und habe stets zwischen 20 und $40 \mathrm{mg}$ Prednisolon pro Tag betragen. Die Verschreibung der Glukokortikosteroide erfolgte jeweils durch unterschiedliche Hausärzte, die der Patient im Laufe der Zeit kontinuierlich wechselte. Der Patient berichtet von einer Gewichtszunahme von $36 \mathrm{~kg}$ in den letzten Jahren. Vor 4 Jahren sei eine Operation am rechten Auge aufgrund einer Katarakt erfolgt, worauf es im Anschluss zu einer Netzhautablösung mit folgender Erblindung rechts gekommen sei. Nach einem Sturz auf die rechte Hand vor 3 Wochen könne er zudem eine Stufenbildung am Handrücken tasten.

\section{Befund}

Ein 28-jähriger Patient in adipösem Ernährungszustand (Body-Maß-Index $35,8 \mathrm{~kg} / \mathrm{m}^{2}$ ) mit Stammfettsucht und nuchaler Fettgewebsvermehrung („Büffelnacken") (• Abb. 1). Im Nackenbereich, an den Oberarmen, abdominell sowie an den Oberschenkeln zeigen sich unscharf begrenzte Erytheme, teilweise mit Exkoriationen und Lichenifikation im Bereich der Beugeseiten. Die befallene Körperoberfläche („body surface area“ [BSA]) beträgt ca. $10 \%$. Im Gesicht zeigt sich eine Plethora. Ausgeprägte Striae distensae finden sich am Rumpf und den Oberarmen (- Abb. 2). An der rechten Hand lässt sich über den Mittelhandknochen eine Stufenbildung tasten. 


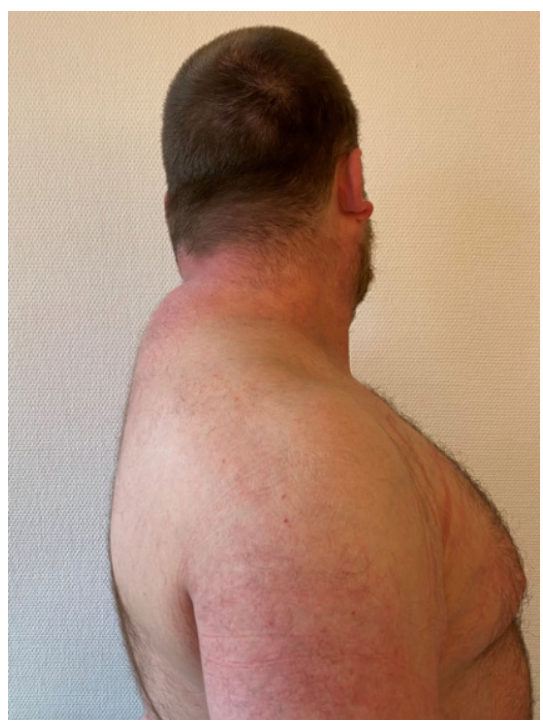

Abb. 1 \ Nuchale Fettgewebsvermehrung; flächige Erytheme nuchal sowie an den Oberarmen

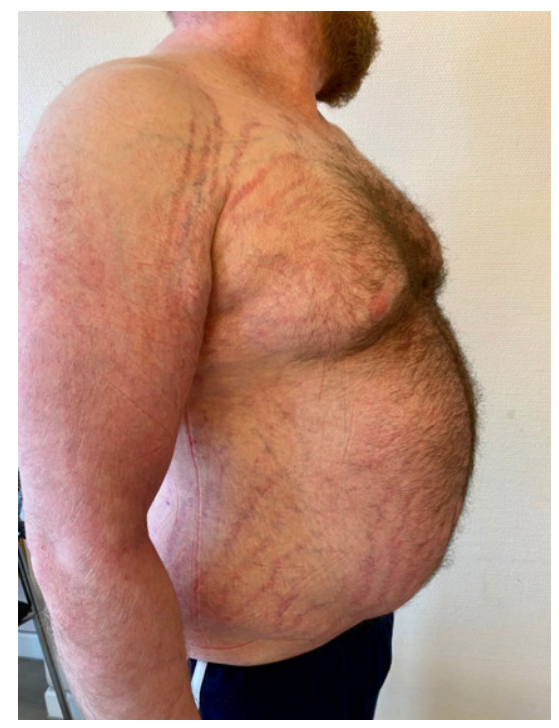

Abb. $2 \triangle$ Striae distensae am Rumpf und an den Oberarmen bei Stammfettsucht

\begin{tabular}{|c|c|}
\hline Nebenwirkung & Häufigkeit \\
\hline Arterielle Hypertonie & $>30 \%$ \\
\hline Knochenfrakturen/Osteoporose & $21-30 \%$ \\
\hline Übelkeit/Erbrechen/andere gastrointestinale Beschwerden & $1-5 \%$ \\
\hline Herzerkrankungen & $4 \%$ \\
\hline Katarakt & $1-3 \%$ \\
\hline Diabetes mellitus Typ 2/hyperglykämische Stoffwechsellage & Bis zu 4-fach häufiger \\
\hline Schlafstörungen & Keine Angabe \\
\hline
\end{tabular}

\section{Diagnostik}

Im Rahmen der Diagnostik der AD zeigten sich ein stark erhöhtes Gesamt-Immunglobulin $E(>5000)$ sowie ein positiver Phadiatop-Test $(\mathrm{S} \times 1)$ auf häufige Inhalationsallergene. Bei einem Eczema Area and Severity Index (EASI) von 27 lag eine schwere $A D$ vor, der Dermatologische-Lebensqualitäts-Index (DLQI) wies bei einem Wert von 20 eine schwere Beeinträchtigung der Lebensqualität auf.

Zur Abklärung der Langzeitschäden unter der systemischen Glukokortikosteroidtherapie erfolgte eine weiterführende Diagnostik. Durch wiederholte Blutdruckmessungen konnte eine arterielle Hypertonie ausgeschlossen werden. Laborchemisch zeigte sich der $\mathrm{HbA} 1 \mathrm{c}$ mit $6,1 \%$ (Referenz 4,8-5,6\%) erhöht. Zudem konnte ein ausgeprägter Vitamin-D-Mangel mit $7 \mu \mathrm{g} / \mathrm{l}$ nachgewiesen werden (Referenz $30-60 \mu \mathrm{g} / \mathrm{l})$. Weitere osteologische Labor- parameter (Kalzium, Phosphat, alkalische Phosphatase [AP], knochenspezifische AP, $\gamma$-Glutamyltransferase, Kreatinin, Osteocalcin und Desoxypyridinolin Crosslinks im Urin) zeigten sich unauffällig.

Die Knochendichtemessung ergab eine spinal betonte Osteoporose mit einem T-Score der Wirbelsäule von -2,8 (Referenz T kleiner als $-2,5$ Standardabweichungen).

Das Röntgenbild der rechten Hand zeigte eine subakute Schaftfraktur des Os metacarpale IV und V. In der Sonographie des Abdomens konnte eine Steatosis hepatis nachgewiesen werden.

Die ophthalmologische Mitbeurteilung ergab einen Normalbefund des linken $\mathrm{Au}$ ges sowie die bereits bekannte Netzhautablösung des rechten Auges.

\section{Therapie und Verlauf}

Im Rahmen des stationären Aufenthaltes wurde die Prednisolon-Therapie mit
$20 \mathrm{mg}$ pro Tag fortgeführt. Vor dem Hintergrund der langjährigen Steroidbehandlung erfolgte unter endokrinologischer Mitbeurteilung die Festlegung eines Ausschleichplans. Dieser sah eine Reduktion von Prednisolon um jeweils $2,5 \mathrm{mg}$ im Abstand von 3 Wochen vor. Zusätzlich solle bei einer Dosis von $5 \mathrm{mg}$ Prednisolon ein ACTH(adrenocorticotropes Hormon)Kurztest zur Untersuchung der endokrinen Funktion der Nebennierenrinde erfolgen. Aufgrund der Gefahr einer Addison-Krise bei zu schneller Reduktion erfolgte eine ambulante endokrinologische Anbindung.

Bei schwerer AD wurde die leitliniengerechte Systemtherapie mit Dupilumab $600 \mathrm{mg}$ als Initialdosis, gefolgt von $300 \mathrm{mg}$ als Erhaltungsdosis subkutan alle 14 Tage eingeleitet. Eine Lichttherapie mittels UVB$311 \mathrm{~nm}$ musste nach 2 Sitzungen mit einer Dosis von jeweils $0,2 \mathrm{~J} / \mathrm{cm}^{2}$ aufgrund des Auftretens einer Dermatitis solaris beendet werden.

Im Rahmen des osteologischen Behandlungsplans erfolgte eine Substitution von Vitamin $D_{3}$ mittels 20.000 IE 1-mal täglich über 2 Wochen sowie eine im Anschluss 2-mal wöchentliche Gabe. Es ist auf eine Aufnahme von $1000 \mathrm{mg}$ Kalzium über die Nahrung zu achten, die im Falle einer nicht ausreichenden Zufuhr durch eine additive Gabe von Calciumglukonat/-citrat ergänzt werden sollte. Durch die Kollegen der Osteologie wurde die Indikation zur spezifischen antiresorptiven Therapie gestellt, die nach erneuter Evaluation des Vitamin D-, Kalzium- und Knochenstoffwechsels unter optimierter Basistherapie vorgesehen ist. Eine engmaschige osteologische Anbindung wurde initiiert. Die subakute Schaftfraktur des Os metacarpale IV und $\mathrm{V}$ wurde konservativ mittels Orthese versorgt. Bei beginnendem Steroiddiabetes erfolgte zudem eine Diätberatung mit Aufklärung über eine gesunde Ernährung sowie geeignetes Bewegungsverhalten.

\section{Diskussion}

In den letzten Jahren ließ sich ein rasanter Fortschritt für die Behandlung von Patienten mit schwerer AD verzeichnen. Neben den bereits zugelassenen Biologika Dupilumab (Interleukin[IL]-4-IL13-Rezeptor-Antikörper) und Tralokinu- 
mab (IL-13-Antikörper) sowie den oralen Januskinase(JAK)-Inhibitoren Baricitinib (JAK1/JAK2) und Upadacitinib (JAK1) stehen zahlreiche weitere Systemtherapeutika kurz vor Markteinführung und werden das Therapiespektrum der AD zusätzlich erweitern [1, 6].

Dennoch haben noch nicht alle Patienten Zugang zu diesen modernen Systemtherapien, wie dieser Fallbericht über einen jungen Patienten unter Langzeitbehandlung mit systemischen Glukokortikosteroiden verdeutlicht.

In $\bullet$ Tab. 1 sind die häufigsten Nebenwirkungen einer langjährigen Therapie mit Glukokortikosteroiden aufgelistet [3]. Der Patient wies bei einem Alter von 28 Jahren bereits zahlreiche dieser Nebenwirkungen auf.

Die Leitlinie für die Behandlung der AD empfiehlt den Einsatz systemischer Glukokortikosteroide lediglich in Ausnahmefällen im Rahmen einer akuten Exazerbation bei expliziter Ablehnung einer Langzeittherapie [4, 5]. Das Management im Rahmen der Versorgung von Patienten mit langjähriger Einnahme von Glukokortikosteroiden ist - wie hier aufgezeigt - komplex und verlangt ein interdisziplinäres Vorgehen.

Leider ist bis heute der Einsatz systemischer Glukokortikosteroide bei Patienten mit AD keine Seltenheit. Gemäß den Daten des deutschen Neurodermitis-Registers TREATgermany erhielt bereits mehr als die Hälfte der Patienten mit schwerer AD orale Glukokortikoide [2]. Insbesondere vor dem Hintergrund der zahlreichen Innovationen für die $A D$ muss es das Ziel sein, möglichst allen Patienten mit einer schweren $A D$ eine leitliniengerechte, moderne Systemtherapie zukommen zulassen. Hierbei kommt der Information von Ärzten und Patienten über die neuen Behandlungsoptionen eine große Bedeutung zu.

\section{Fazit für die Praxis}

- Die Zulassung moderner Systemtherapien erlaubt heute auch für die schwere atopische Dermatitis eine sehr wirksame und zudem auch sichere und gut verträgliche Behandlung.

- Eine Anwendung systemischer Glukokortikosteroide bei atopischer Dermatitis ist

\section{Inappropriate systemic therapy in severe atopic dermatitis-severe long-term damage}

The therapeutic options for treatment of severe cases of atopic dermatitis have been limited until recently, but fundamentally improved with the approval of the first biological dupilumab at the end of 2017. With the biological tralokinumab and the Janus kinase inhibitors baricitinib and upadacitinib, further new systemic drugs have recently been approved. Nevertheless, there are cases in which modern treatment options are not taken into account, as shown by a 28-year-old patient with serious side effects from long-term treatment with systemic glucocorticoids. In addition to the extensive clarification of the consequential damage, guideline-based therapy with dupilumab was initiated as well as interdisciplinary cooperation with endocrinologists, ophthalmologists, osteologists and nutritionists.

\section{Keywords}

Atopic dermatitis · Systemic therapy · Oral glucocorticoids · Adverse events

nur in Ausnahmefällen und immer nur über kurze Dauer angezeigt.

- Im Rahmen der Diagnostik und Versorgung von Patienten mit langjähriger Einnahme von Glukokortikosteroiden ist ein interdisziplinäres Vorgehen von Bedeutung.

\section{Korrespondenzadresse}

\section{Dr. med. F. Abeck}

Klinik und Poliklinik für Dermatologie und Venerologie, Universitätsklinikum HamburgEppendorf

Martinistraße 52, 20246 Hamburg, Deutschland finn.abeck@uke.de

Funding. Open Access funding enabled and organized by Projekt DEAL.

\section{Einhaltung ethischer Richtlinien}

Interessenkonflikt. F. Abeck, N. Booken und S.W. Schneider geben an, dass kein Interessenkonflikt besteht.

Für diesen Beitrag wurden von den Autoren keine Studien an Menschen oder Tieren durchgeführt. Für die aufgeführten Studien gelten die jeweils dort angegebenen ethischen Richtlinien. Für Bildmaterial oder anderweitige Angaben innerhalb des Manuskripts, über die Patienten zu identifizieren sind, liegt von ihnen und/oder ihren gesetzlichen Vertretern eine schriftliche Einwilligung vor.

Open Access. Dieser Artikel wird unter der Creative Commons Namensnennung 4.0 International Lizenz veröffentlicht, welche die Nutzung, Vervielfältigung, Bearbeitung, Verbreitung und Wiedergabe in jeglichem Medium und Format erlaubt, sofern Sie den/die ursprünglichen Autor(en) und die Quelle ordnungsgemäß nennen, einen Link zur Creative Commons Lizenz beifügen und angeben, ob Änderungen vorgenommen wurden.

Die in diesem Artikel enthaltenen Bilder und sonstiges Drittmaterial unterliegen ebenfalls der genannten Creative Commons Lizenz, sofern sich aus der Abbildungslegende nichts anderes ergibt. Sofern das betreffende Material nicht unter der genannten Creative Commons Lizenz steht und die betreffende Handlung nicht nach gesetzlichen Vorschriften erlaubt ist, ist für die oben aufgeführten Weiterverwendungen des Materials die Einwilligung des jeweiligen Rechteinhabers einzuholen.

Weitere Details zur Lizenz entnehmen Sie bitte der Lizenzinformation auf http://creativecommons.org/ licenses/by/4.0/deed.de.

\section{Literatur}

1. Bieber T (2021) Atopic dermatitis: an expanding therapeutic pipeline for a complex disease. Nat Rev Drug Discov. https://doi.org/10.1038/s41573021-00266-6

2. Heratizadeh A, HaufeE, Stölzl Detal(2020) Baseline characteristics, disease severity and treatment history of patients with atopic dermatitis included in the German AD Registry TREATgermany. J Eur Acad Dermatol Venereol 34:1263-1272

3. Rice JB, White AG, Scarpati LM et al (2017) Longterm systemic corticosteroid exposure: a systematic literature review. Clin Ther 39:2216-2229

4. Werfel T, Heratizadeh A, Aberer W et al (2016) S2k guideline on diagnosis and treatment of atopic dermatitis—short version. Allergo J Int 25:82-95

5. Wollenberg A, Barbarot S, Bieber T et al (2018) Consensus-based European guidelines for treatment of atopic eczema (atopic dermatitis) in adults and children: part II. J Eur Acad Dermatol Venereol 32:850-878

6. Worm M, FrancuzikW, Kraft Metal (2020) Moderne Therapie der atopischen Dermatitis: Biologika und kleinmolekulare Medikamente. J Dtsch Dermatol Ges 18:1085-1093 